\title{
Ac-SDKP Attenuates Activation of Lung Macrophages and Bone Osteoclasts in Rats Exposed to Silica by Inhibition of TLR4 and RANKL Signaling Pathways
}

Fuyu Jin, (D)' Fei Geng, ${ }^{2}$ Dingjie $\mathrm{Xu},{ }^{3}$ Yaqian $\mathrm{Li}$, (D) $^{1}$ Tian Li,' Xinyu Yang, (D) Shupeng Liu, (D) 'Hui Zhang,' Zhongqiu Wei, ${ }^{1}$ Shifeng $\mathrm{Li}^{2}$ Xuemin Gao, ${ }^{2}$ Wenchen $\mathrm{Cai}^{2}{ }^{2} \mathrm{Na} \mathrm{Mao,}{ }^{2}$ Xue $\mathrm{Yi}^{4}{ }^{4}$ Heliang Liu, ${ }^{2}$ Ying Sun, ' Fang Yang, (D) ${ }^{2}$ Hong $\mathrm{Xu} \mathbb{1 D}^{1,2}$

'Basic Medical College, Hebei Key Laboratory for Chronic Diseases, North China University of Science and Technology, Tangshan, Hebei Province, 0632 I0, People's Republic of China; ${ }^{2}$ School of Public Health, Hebei Key Laboratory for Organ Fibrosis Research, North China University of Science and Technology, Tangshan, Hebei Province, 063210, People's Republic of China; ${ }^{3}$ Traditional Chinese Medicine College, North China University of Science and Technology, Tangshan, Hebei Province, 063210, People's Republic of China; ${ }^{4}$ Key Laboratory of Functional and Clinical Translational Medicine, Xiamen Medical College, Xianmen, Fujian Province, 361023, People's Republic of China

Correspondence: Hong $\mathrm{Xu}$ 2I Bohai Road, Caofeidian District, Tangshan, Hebei Province, 063210,

People's Republic of China

Tel +86 315-88I6236

Email xuhong@ncst.edu.cn
Background: Silica-induced inflammatory activation is associated with silicosis and various non-respiratory conditions. The present study was designed to examine the anti-inflammatory effects of N-acetyl-seryl-aspartyl-lysyl-proline (Ac-SDKP) on lung macrophages and bone osteoclasts after silica inhalation in rats.

Methods: Wistar rats and NR8383 and RAW 264.7 cell lines were used in the present study. The receptor activator of nuclear factor kappa-B ligand (RANKL) and toll-like receptor 4 (TLR4) signaling pathways was measured in the lung tissue of rats or NR8383/RAW 264.7 cells exposed to silica. The microarchitecture of the trabecular bone in the tibia and femur was evaluated in silicotic rats. Furthermore, the roles of Ac-SDKP on silicotic rats, silicatreated NR8383/RAW 264.7 cells, and RANKL-induced osteoclast differentiation were studied.

Results: The data indicated that silica inhalation might activate the RANKL and TLR4 signaling pathways in lung macrophages, thus inducing the lung inflammatory and proteolytic phenotype of macrophages and osteoclasts in lung and bone. Ac-SDKP maintained the lung elastin level by inhibiting lung inflammation and macrophage activation via the RANKL and TLR4 signaling pathways. Ac-SDKP also attenuated the reduction in femoral bone mineral density in silicotic rats by inhibiting osteoclast differentiation via the RANKL signaling pathway.

Conclusion: Our findings support the hypothesis that inhalation of crystalline silica induces activation of lung macrophages and bone osteoclasts via the RANKL and TLR4 signaling pathways. Ac-SDKP has the potential to stabilize lung homeostasis and bone metabolism.

Keywords: silicosis, N-acetyl-seryl-aspartyl-lysyl-proline, macrophage, osteoclast

\section{Introduction}

Inhalation of silica in the occupational environment is a major cause of silicosis, and phagocytosis of these particles by macrophages initiates an inflammatory response. ${ }^{1}$ Apart from silicosis, silica-induced inflammatory activation induces the development of various respiratory and non-respiratory conditions including silicosis, autoimmune disease, sarcoidosis, lung cancer, pulmonary infections, and renal disease. ${ }^{2-5}$

Osteoporosis has debilitating systemic effects in patients with silicosis, ${ }^{6}$ horses with silicate-associated osteoporosis, ${ }^{7}$ and rats that have inhaled silica. ${ }^{8}$ Our 
previous study showed that low bone mineral density (BMD) can occur independently of established silicosisosteoporosis risk factors such as age, nutrition, medical treatment, and immobility. ${ }^{8}$ This suggests that lung inflammation is an independent risk factor for BMD loss. An in vitro study showed that silica activates toll-like receptor 4 (TLR4) signaling to release tumor necrosis factor- $\alpha$ (TNF- $\alpha$ ), interleukin-1 $\beta$ (IL-1 $\beta$ ), and IL-6 in U-937differentiated macrophages. ${ }^{9}$ Considering these previous findings, we hypothesized that inhalation of silica can promote chronic inflammation and lung fibrosis, disrupt bone metabolism, and promote increased bone loss.

Furthermore, lung macrophages exhibit patterns similar to those of bone osteoclasts because they have a common origin from the monocyte lineage and therefore participate in common signaling pathways. ${ }^{10}$ Osteoclast differentiation is characterized by increased bone absorbability and positive expression of tartrate-resistant acid phosphatase (TRAP), and it is regulated by several signaling pathways: receptor activator of nuclear factor kappa-B ligand (RANKL), osteoclast stimulatory transmembrane protein (OC-STAMP), nuclear factor-activated T-cell cytoplasmic 1 (NFATc1), activator protein-1 (AP-1), and nuclear factor kappa-B (NF-kB) signaling pathways. ${ }^{11,12}$ Positivity of TRAP in alveolar macrophages is also found in the lungs of patients with chronic obstructive pulmonary disease and asthma compared with controls, and high TRAP activity is observed in the lungs of mice with experimental chronic obstructive pulmonary disease or asthma. ${ }^{13}$ TRAP-positive macrophages produce various matrix metalloproteinases (MMPs) such as MMP-2 (gelatinase A), MMP-9 (gelatinase B), MMP-12 (macrophage metalloelastase), and MMP-14 (MT1-MMP). ${ }^{14,15}$ Therefore, TRAP-positive lung macrophages might display some molecular signatures of osteoclast differentiation regulated by the RANKL signaling pathway.

Preclinical studies have shown that the naturally occurring tetrapeptide N-acetyl-seryl-aspartyl-lysyl-proline (Ac-SDKP) has greatly beneficial effects with respect to modulation of inflammatory activities in heart, kidney, brain, ${ }^{16}$ and lung injury. ${ }^{17,18}$ Evidence from clinical trials suggests that AcSDKP exerts anti-inflammatory, anti-fibrotic, and proangiogenic potential and is well tolerated in humans. ${ }^{16}$ The anti-inflammatory effects of Ac-SDKP on macrophages indicate that Ac-SDKP may have a potential role in activation of lung macrophages or osteoclast differentiation. ${ }^{19,20}$ Although the effect of Ac-SDKP on fibrosis disorders has been well documented, the effects of Ac-SDKP on bone metabolism and related signaling pathways remain unknown.

Therefore, we hypothesized that inhalation of silica may activate the inflammation-related TLR4 and RANKL signaling pathways in lung macrophages to enhance a proteolytic phenotype of macrophages in the lung and activate osteoclast differentiation in bone, which can be attenuated by treatment with Ac-SDKP.

\section{Methods}

\section{Establishment of Silicosis Disease Model}

Three-week-old male Wistar rats were purchased from Vital River Laboratory Animal Technology Co. Ltd. (SCXY 2016-0008; Beijing, China). Upon arrival, the rats were maintained in a 12-h/12-h light/dark cycle and provided with food and water ad libitum. The silicotic model was established by a HOPE MED 8050 exposure control apparatus (HOPE Industry and Trade Co. Ltd., Tianjin, China) as reported previously. ${ }^{8,18}$ The study protocol was approved by the Committee on the Ethics of North China University of Science and Technology (LX2019033) and complied with the US National Institutes of Health Guide for the Care and Use of Laboratory Animals. ${ }^{21}$

The rats were allocated into three groups as follows: 1) control group: inhalation of pure air for 16 weeks and then treatment with $0.9 \%$ saline for another 8 weeks; 2) silicosis group: inhalation of $50 \pm 10 \mu \mathrm{g} / \mathrm{m}^{3}$ of silica (s5631; Sigma-Aldrich, St. Louis, MO, USA; ground and then heated at $180^{\circ} \mathrm{C}$ for $6 \mathrm{~h}$ ) for 16 weeks and then treatment with $0.9 \%$ saline for another 8 weeks; and 3) Ac-SDKP treatment group: inhalation of silica for 16 weeks and then treatment with $800 \mu \mathrm{g} / \mathrm{kg} /$ day of Ac-SDKP for another 8 weeks. ${ }^{18}$ Bone samples were decalcified in $15 \%$ EDTA$\mathrm{Na}_{2}(\mathrm{pH} 7.25)$ at $4^{\circ} \mathrm{C}$ for 6 to 8 weeks. The samples were sequentially dehydrated, embedded in paraffin, and cut into 8 - $\mu \mathrm{m}$-thick sections for staining.

\section{Cell Culture and Treatments}

NR8383 alveolar macrophages and the RAW 264.7 murine monocyte/macrophage cell line were obtained from the Chinese Academy of Sciences cell library (Shanghai, China) and cultured in Ham's F-12K medium (J120703; Shanghai BasalMedia Technologies Co., Ltd. Shanghai, China). Cells were plated in $25-\mathrm{cm}^{2}$ flasks and treated with or without one or more of the following: $50 \mu \mathrm{g} / \mathrm{cm}^{2}$ silica, ${ }^{22} 30 \mathrm{ng} / \mathrm{mL}$ recombinant mouse RANKL (462-TEC -010; R\&D Systems, Minneapolis, MN, USA), ${ }^{23} 10 \mathrm{nmol} /$ 
L Ac-SDKP (H-1164; Bachem AG, Torrance, CA, USA), ${ }^{18} 10 \mathrm{nmol} / \mathrm{L}$ Ac-ADKP (substitution of Ser by Ala in Ac-SDKP) (PCM10745-0912; Pepmic, Suzhou, China), ${ }^{24}$ and $10 \mu \mathrm{mol} / \mathrm{L}$ TLR-C34 (18512; Cayman Chemical, Ann Arbor, MI, USA). ${ }^{25}$

\section{TRAP Staining}

The presence of TRAP was assessed using a histochemical method that employs conversion of chromogen Fast Red by active TRAP. ${ }^{13}$ Lung multinucleated giant cells and bone osteoclasts were identified by three or more nuclei. The number of TRAP-positive cells was counted manually with the aid of Image-Pro Plus Version 6.0.0.260 (Media Cybernetics, Inc., Rockville, MD, USA).

\section{Alkaline Phosphatase (ALP) Staining}

The sections were stained with a BCIP/NBT kit for ALP activity. Quantitative calculation of osteoblasts was manually performed with the aid of Image-Pro Plus Version 6.0.0.260 (Media Cybernetics, Inc.).

\section{Immunohistochemistry and Immunofluorescence Staining}

Paraffin-embedded sections were deparaffinized and rehydrated, and endogenous peroxidases were quenched with $3 \%$ hydrogen peroxide for $15 \mathrm{~min}$. The samples were then incubated with primary antibodies to RANK (A13382; ABclonal Biotechnology, Woburn, MA, USA), RANKL (ARG65870; arigo Biolaboratories, Taiwan, China), TLR4 (ARG20515; arigo Biolaboratories), TNF- $\alpha$ (GTX110520; GeneTex Inc., Irvine, CA, USA), and MMP-12 (ET1602-42; HuaAn Biotechnology Co. Ltd., Zhejiang, China) at a dilution of 1:200 overnight at $4^{\circ} \mathrm{C}$, followed by incubation with secondary antibodies (PV6000; Beijing Zhongshan Jinqiao Biotechnology Co. Ltd., Beijing, China) at $37^{\circ} \mathrm{C}$ for $20 \mathrm{~min}$. Immunoreactivity was visualized using DAB (ZLI-9018; Beijing Zhongshan Jinqiao Biotechnology Co. Ltd.). Brown staining was considered positive. ${ }^{8}$

For immunofluorescence staining, the samples were incubated with RANK/CD68 (a marker of macrophages), RANKL/CD68, TNF- $\alpha / C D 68$, and TLR4/CD68 at a dilution of 1:200 overnight at $4^{\circ} \mathrm{C}$, followed by incubation with donkey anti-rabbit TRITC secondary antibody and donkey anti-mouse FITC highly cross-adsorbed secondary antibody (A16028 and A16018; Novex, Life Technologies, Frederick, MD, USA) at $37^{\circ} \mathrm{C}$ for $40 \mathrm{~min}$.
The cell samples were incubated with TLR4 at a dilution of 1: 200 overnight at $4^{\circ} \mathrm{C}$, followed by incubation with donkey anti-rabbit FITC secondary antibody (A16024, Novex). Nuclei were stained with ProLong Antifade Reagent with DAPI (8961s; Cell Signaling Technology, Inc., Danvers, MA, USA).

\section{Osteoclast Resorption Pit Assay}

A 24-well Osteo Assay Plate (3987; Corning, Kennebunk, ME, USA) was used to evaluate the bone resorptive activities of osteoclasts. Cells were removed with 5\% sodium hypochlorite for $5 \mathrm{~min}$, and the pit area was observed and analyzed by Image-Pro Plus software.

\section{Subchondral Bone Microstructure Measurements}

The proximal tibia and distal femur were scanned by a SkyScan 1176 scanner micro-computed tomography system (Bruker, Kontich, Belgium) to quantify the microarchitecture of the trabecular bone. ${ }^{8}$ Both regions of interest of the tibia and femur were located under the epiphyseal plate at the cross-sectional level, which excluded the cortical shell. The following morphometric parameters were calculated using software developed for the machine to describe the bone mass and structure: $\mathrm{BMD}$, bone volume to total volume fraction, trabecular number, trabecular thickness, trabecular separation, structure model index, trabecular pattern factor, and degree of anisotropy.

\section{Western Blot Analysis}

Western blotting was performed as described previously. ${ }^{18}$ Primary antibodies included polyclonal rabbit antibodies to RANK, RANKL, OC-STAMP (2051. PB1; FabGennix Inc., Frisco, TX, USA), AP-1 (ARG65852; arigo Biolaboratories Corp.), NFATc1 (DF6446; Affinity Biosciences, Cincinnati, OH, USA), NF-KB (ARG51013; arigo Biolaboratories Corp.), TLR4 (ARG20515; arigo Biolaboratories Corp.), myeloid differentiation primary response protein 88 (MyD88) (ab2068; Abcam, Cambridge, UK), p-IкB $\mathrm{B}^{\mathrm{Ser} 32 / \mathrm{Ser} 36}$ (AF2002; Affinity Biosciences), TNF- $\alpha$, MMP-12, $\alpha$ tubulin (AF7010, Affinity Biosciences), and $\beta$-actin (AC026, ABclonal Biotechnology) at a dilution of 1:1000. After three washes and incubation with goat antirabbit or anti-mouse secondary antibodies (074-1506/ 074-1806; Kirkegaard \& Perry Laboratories, Gaithersburg, MD, USA) at a dilution of 1:5000 in 
blocking buffer, immunoblots were visualized using ECL prime Western blotting detection reagent (ZD310A; ZomanBio, Beijing, China). The results were normalized against the $\alpha$-Tub expression level and corresponding control.

\section{Enzyme-Linked Immunosorbent Assay}

The serum concentrations of TNF- $\alpha$ and RANKL were determined with enzyme-linked immunosorbent assay kits (RK00029; ABclonal and ARG81958, arigo Biolaboratories Corp.). The minimal detectable concentrations of TNF- $\alpha$ and RANKL for these assays were $31.2 \mathrm{pg} /$ $\mathrm{mL}$ and $15.625 \mathrm{pg} / \mathrm{mL}$, respectively.

\section{Statistical Analysis}

Statistical analyses were performed using SPSS 20.0 software (IBM Corp., Armonk, NY, USA). Two-group comparisons were made using the unpaired Student's $t$-test, and multiple-group comparisons were made using oneway analysis of variance followed by Tukey's post-hoc test. Statistical significance was achieved when $P<0.05$ at a $95 \%$ confidence interval.

\section{Results}

\section{Ac-SDKP Reduces Formation of} TRAP-Positive Macrophages, Inflammation, and Activation of RANKL Signaling in Rats Exposed to Silica

Previous research has established that Ac-SDKP treatment reduces the formation of silicotic granulomas, myofibroblast differentiation, and collagen deposition and improves pulmonary function. ${ }^{8,18,26}$ In the present study, mononuclear and multinuclear TRAP-positive macrophages were observed in silicotic rats, and treatment with Ac-SDKP

reduced the number of TRAP-positive macrophages (Figure 1). Consistent with a previous study, ${ }^{27}$ positive expression of RANK and RANKL was observed in lung macrophages and within silicotic lesions (Figure 2A and B; Supplementary Figure 1). As shown in Figure 2C, Ac-SDKP prevented activation of RANK, RANKL, OC-STAMP, AP1 , NFATc1, and NF- $\mathrm{kB}$ in rats exposed to silica.

Furthermore, expression of TLR4 and TNF- $\alpha$ was observed in lung macrophages and silicotic lesions (Figure $3 \mathrm{~A}$ and B; Supplementary Figure 2). Treatment with AcSDKP attenuated the increased levels of TLR4, MyD88, p-IкB, and TNF- $\alpha$ in rats that had inhaled silica (Figure 3C). Furthermore, Ac-SDKP treatment reduced the high levels of serum TNF- $\alpha$ and RANKL in silicotic rats (Figure 3D).

Elastin staining revealed a protective effect of Ac-SDKP on stabilization of elastin in rats that had inhaled silica (Figure 4A). Ac-SDKP also attenuated the elevation of MMP-12 in silicotic rats (Figure 4B; Supplementary Figure 3A). The co-location of TRAP, RANK, and MMP-12 in lung macrophages is shown in Supplementary Figure 3B.

\section{Ac-SDKP Reduces TRAP-Positive Cells and RANKL Signaling in Macrophages Treated with Silica}

Tissue-resident alveolar macrophages and monocyte-derived macrophages are the dominant macrophage subsets in maintaining lung homeostasis and regulating the inflammatory response in silicosis. ${ }^{28}$ We used NR8383 rat alveolar macrophages to explore the anti-inflammatory effect of Ac-SDKP. As shown in Figure 5, silica promoted an increase in TRAPpositive cells and activated RANKL, RANK, OC-STAMP, AP-1, NFATc1, and NF- $\mathrm{kB}$ expression as well as increase of TLR4, MyD88, p-IкB, TNF- $\alpha$, and MMP-12 in NR8383 macrophages. Treatment with Ac-SDKP reversed all of the silica-induced effects, whereas Ac-ADKP had no effects.
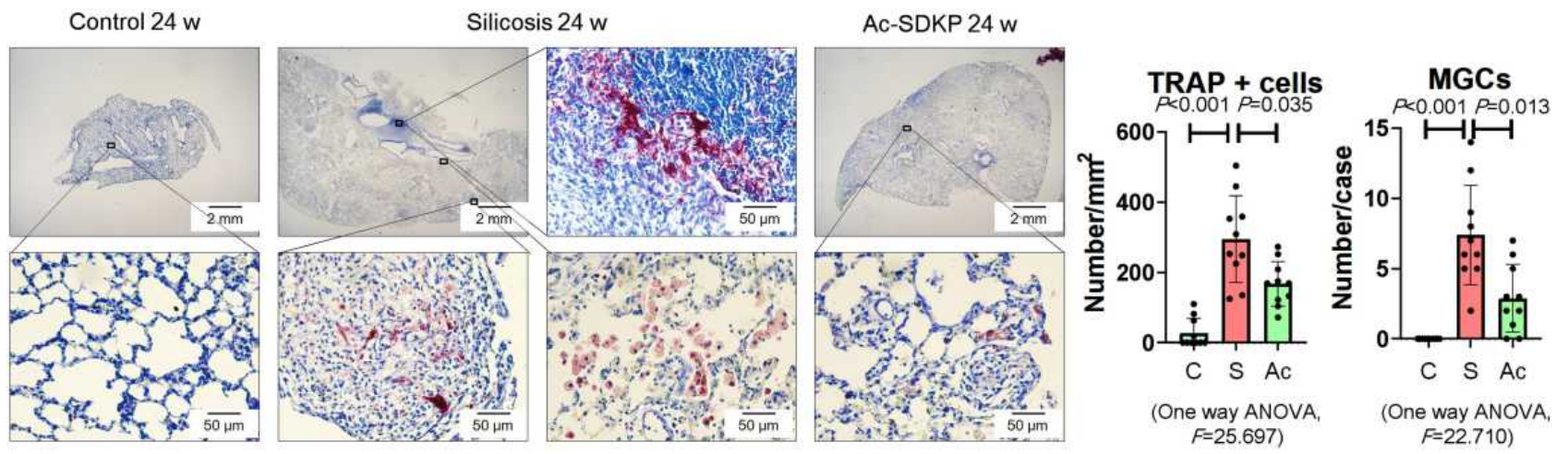

Figure I Ac-SDKP reduces formation of TRAP-positive macrophages. TRAP staining, Bar $=2 \mathrm{~mm}$ and $500 \mu \mathrm{m}$, Data are presented as the mean \pm SD. $\mathrm{n}=10$ per group. 

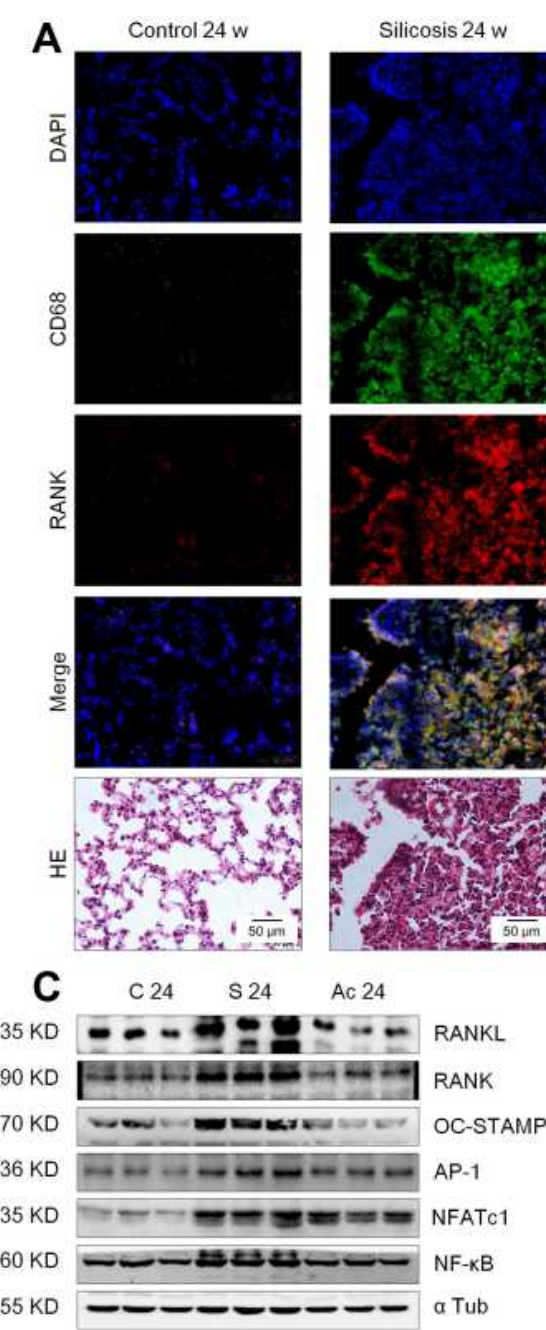

Silicosis 24 w
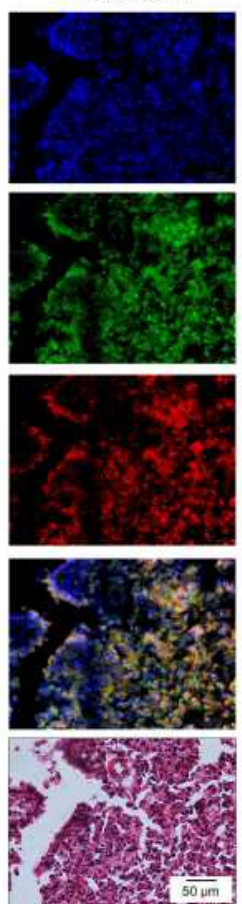

\section{(1)}
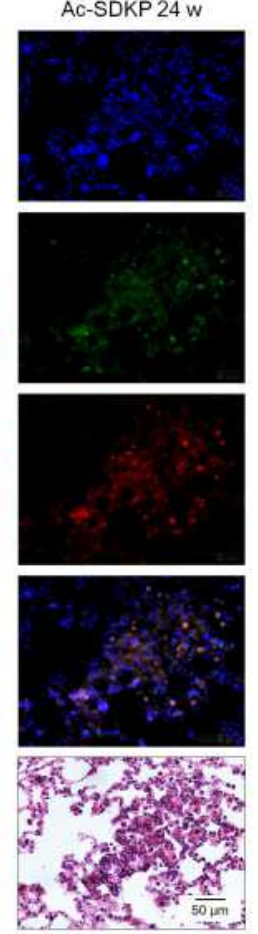

RANKL

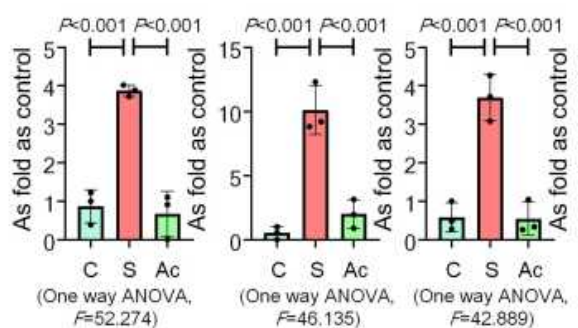

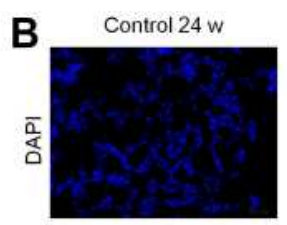
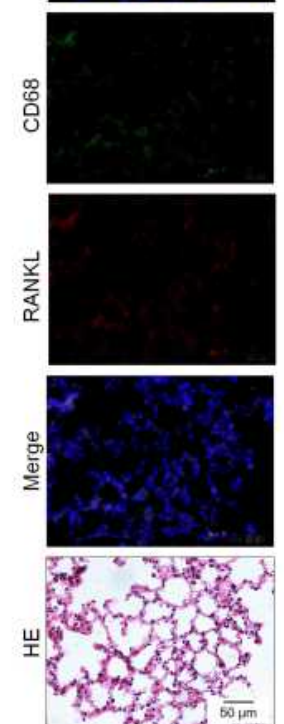

RANK
Silicosis 24 w
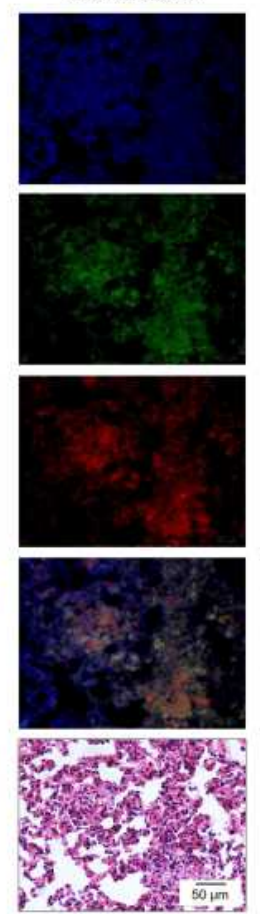

AP-1

$P<0.001$ P $<0.001 \quad P<0.001 \quad P=0.003 \quad P<0.001$ P $<0.001$

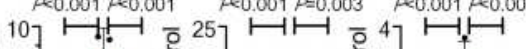

Figure 2 Ac-SDKP reduces activation of RANKL signaling in rats exposed to silica. (A) Co-expression of RANK/CD68 in rats exposed to silica, Bar=50 $\mu$ m; $(\mathbf{B})$ Coexpression of RANKL/CD68 in rats exposed to silica, Bar $=50 \mu \mathrm{m}$; (C) Levels of RANKL, RANK, OC-STAMP, AP-I, NFATcl, and NF- $\kappa B$ in rats lungs measured by Western blotting. Data are presented as the mean \pm SD. $n=3$ per group.

RAW 264.7 cells are commonly used as macrophages in many studies to explore the role of macrophage activation and lung inflammation..$^{29-31}$ In the present study, we found that treatment with silica increased TRAP-positive cells but hardly promoted formation of osteoclast-like cells (Figure 6A). Silica also increased the levels of OC-STAMP, AP-1, NFATc1, and NF- $\kappa B$ in RAW 264.7 cells in a dosedependent manner (Figure 6B). Treatment with AcSDKP reduced TRAP-positive cells and inhibited the major regulators of RANKL signaling pathways (Figure 6C). Furthermore, Ac-SDKP treatment reduced the increased levels of TLR4, MyD88, p-IкB, TNF- $\alpha$, and MMP-12 in silica-treated macrophages, exerting an effect similar to that of the TLR-4 inhibitor TLR4-C34 (Figure 7).

\section{Ac-SDKP Inhibits Osteoclasts in Silicotic Rats}

Consistent with our previous report, ${ }^{8}$ we found that inhalation of silica for 24 weeks induced a significant decrease in BMD in the femur and tibia (Figure 6) of rats. Microarchitectural data also showed changes in the bone trabecular structure in silicotic rats (Figure 8). No obvious lesions were found in the cartilage of the femur or tibia (Supplementary Figure 3C), indicating that the major pathological change in rats exposed to silica was the decrease in BMD. The increase in osteoclasts marked by TRAP staining and the decrease in osteoblasts stained by ALP in the femur and tibia further demonstrated the imbalance in bone homeostasis (Figure 9). To determine whether Ac-SDKP attenuated the decrease in BMD in silicotic rats, we administered Ac-SDKP to rats for 8 
A Control $24 \mathrm{w}$
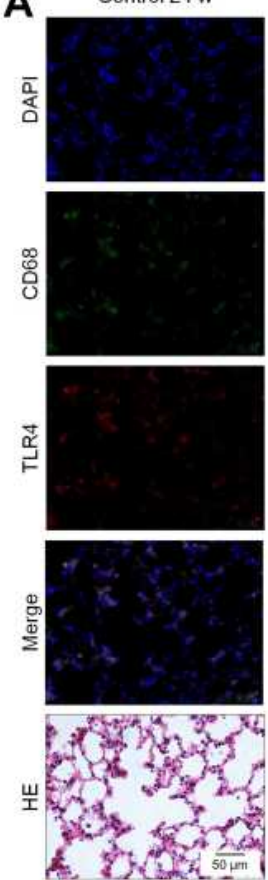

Silicosis $24 \mathrm{w}$
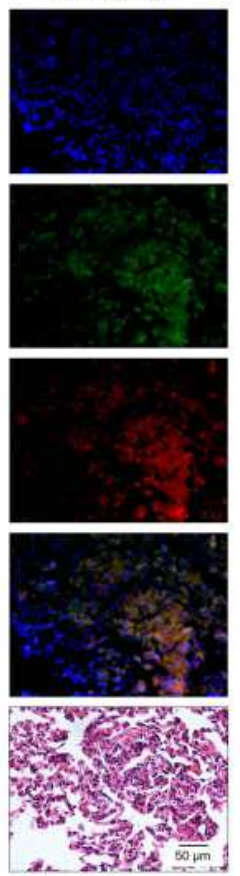

AC-SDKP 24 w
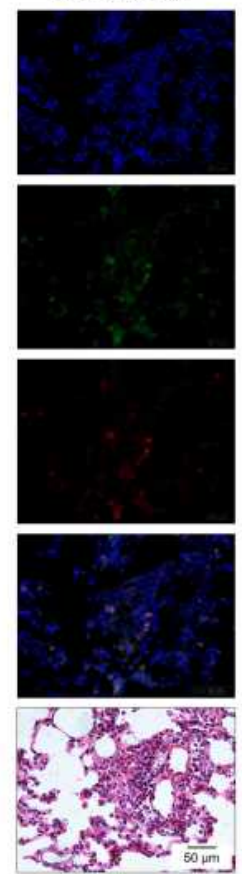

C

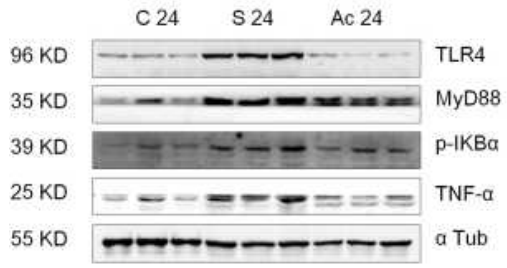

TLR4
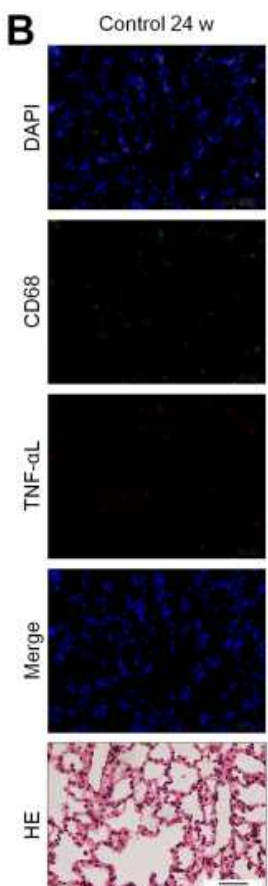

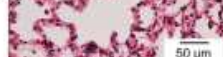

$\mathrm{p}-\mathrm{I} \mathrm{k} \mathrm{B} \alpha$
Silicosis 24 w
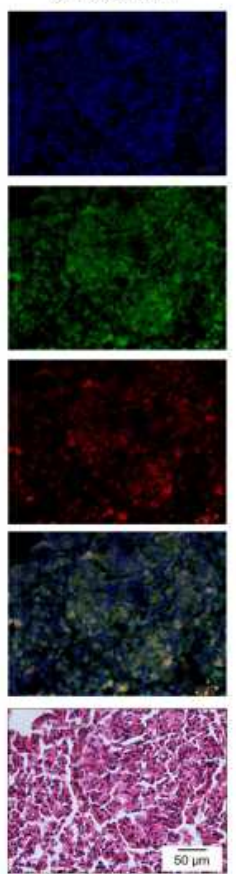

TNF- $\alpha$
Ac-SDKP 24 w
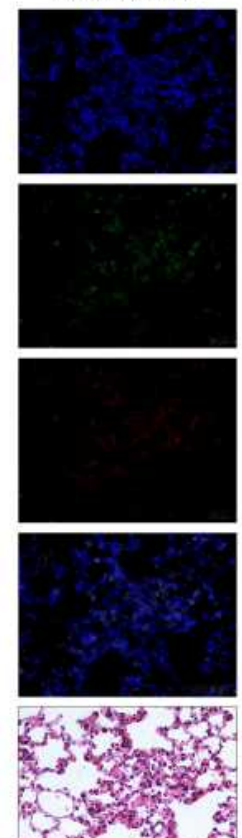
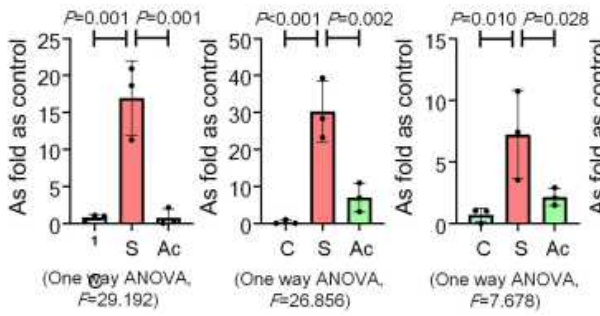

$F=7.678)$

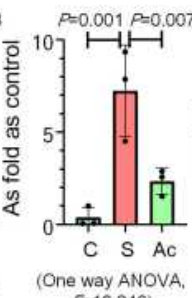

D TNF $-\alpha$

RANKL

Figure 3 Ac-SDKP reduces lung inflammation and activation of TLR4 signaling in rats exposed to silica. (A) Co-expression of TLR4/CD68 in rats exposed to silica, Bar=50 $\mu$; $(\mathbf{B})$

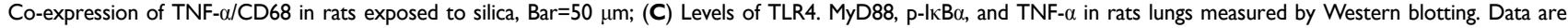
presented as the mean \pm SD. $n=3$ per group. (D) Levels of TNF- $\alpha$ and RANKL in serum of rats measured by ELISA. Data are presented as the mean \pm SD. $n=10$ per group.

A

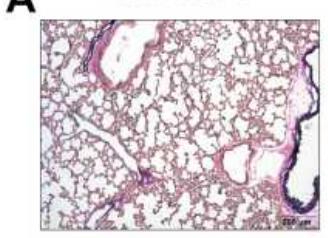

空

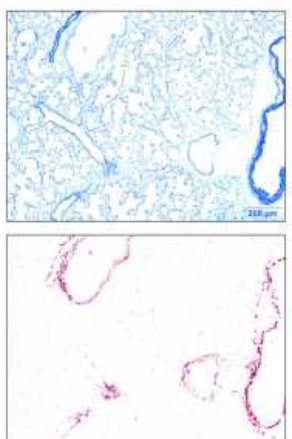

Silicosis $24 \mathrm{w}$
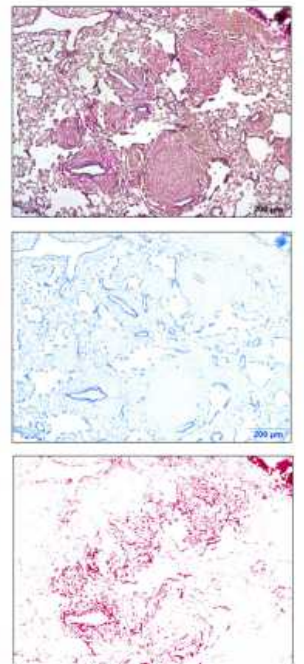

Ac-SDKP $24 \mathrm{w}$

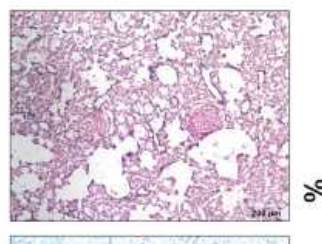

Figure 4 Ac-SDKP inhibits MMP- 12 and stabilizes elastin in silicotic rats. $(\mathbf{A})$ Elastin staining, Bar $=200 \mu m$, Data are presented as the mean \pm SD. $n=8$ per group; $(\mathbf{B})$ Protein expression of MMP-12 in rats lungs measured by Western blotting. Data are presented as the mean \pm SD. $n=3$ per group. 

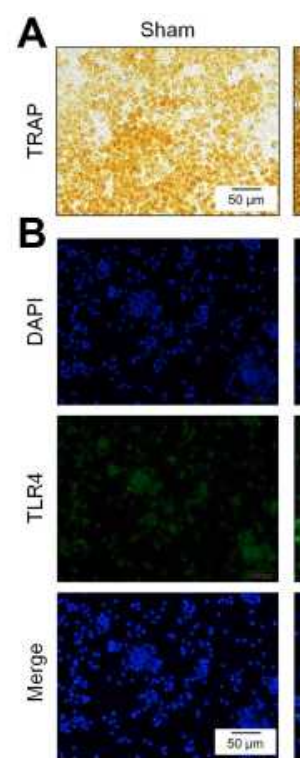

RANKL

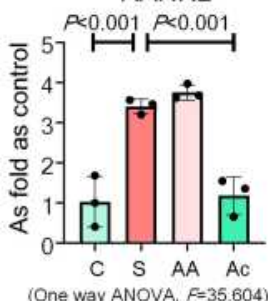

(One way ANOVA, $F=35.604$ )

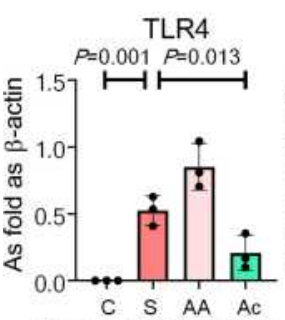

(One way ANOVA, $F=28.187$ )
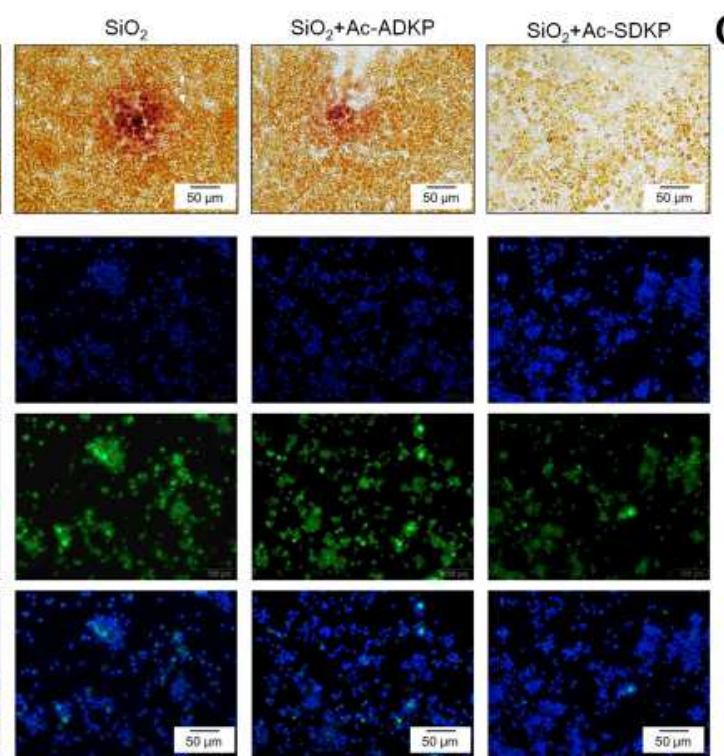

RANK
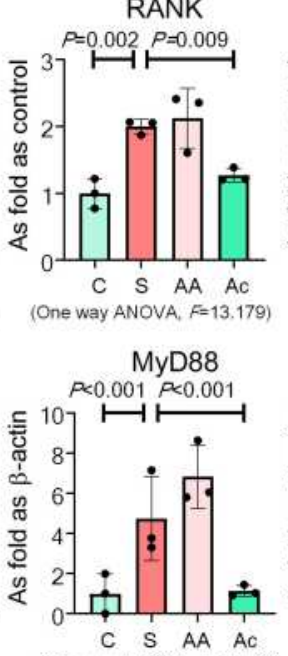

(One way ANOVA, $F=12.402$ )
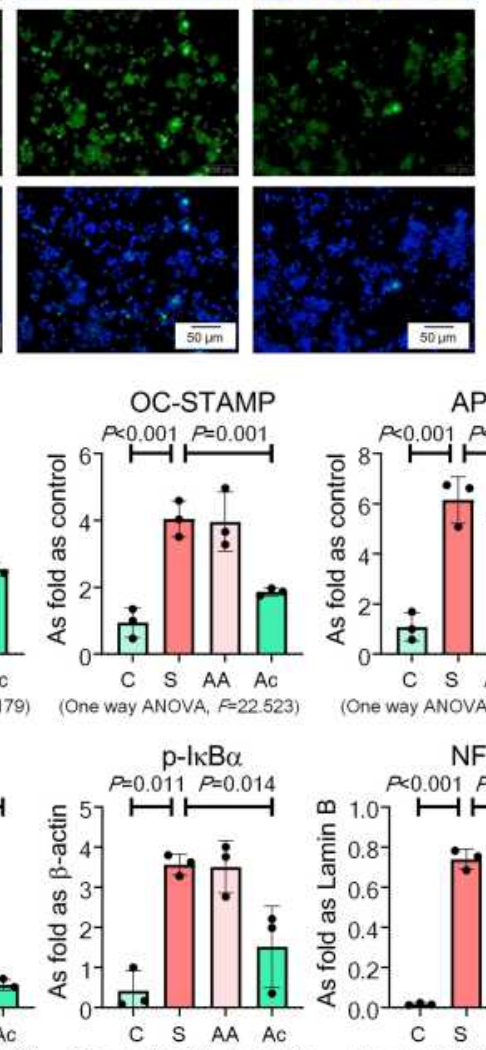

$\overline{50 \mathrm{~mm}}$

AP-1

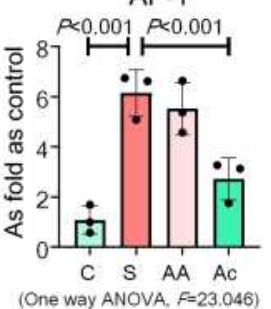

(One way ANOVA, $F=23.046$ )

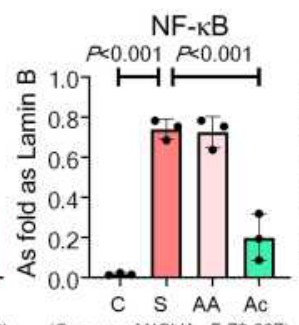

(One way ANOVA, $F=16.178$ ) (One way ANOVA, $F=73.687$ )
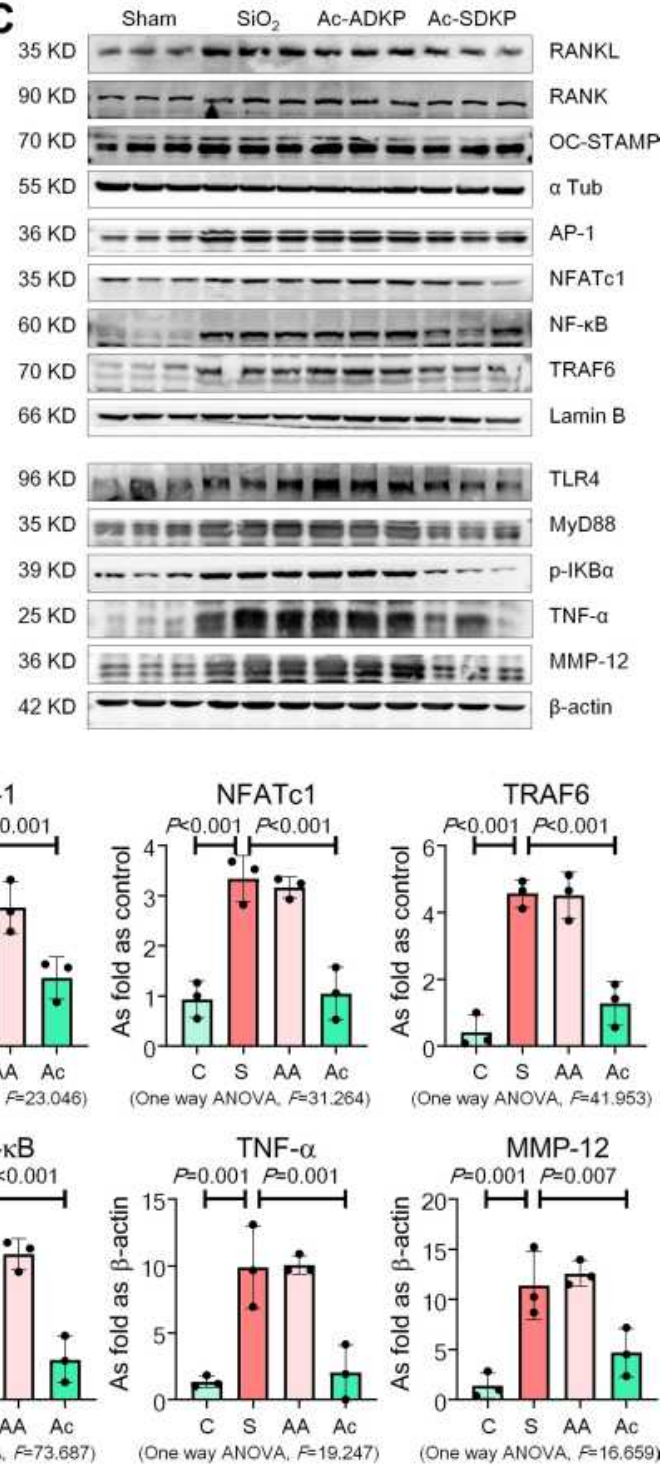

Figure 5 Ac-SDKP inhibits TLR4 and RANKL signaling in NR8383 cells treated with $\mathrm{SiO}_{2 .}$ (A) TRAP staining, Bar= $50 \mu \mathrm{m}$; (B) IF staining of TLR4, Bar=I00 $\mu$ m; (C) Protein expression of RANKL, RANK, OC-STAMP, AP-I, NFATcI, TRAF6, NF- KB, TLR4, MyD88, P-IKB $\alpha$, TNF- $\alpha$, and MMP- 12 in NR8383 cells treated with SiO 2 and Ac-SDKP or not. Data are presented as the mean \pm SD. $n=3$ per group.

weeks after exposure to silica for 16 weeks. We found that treatment with Ac-SDKP attenuated the reduction in BMD of the femur but not in the tibia (Figure 8); however, AcSDKP suppressed the increase in osteoclasts in both the femur and tibia and increased the reduction in osteoblasts induced by inhalation of silica (Figure 9).

\section{Ac-SDKP Inhibits Osteoclast Differentiation of RAW 264.7 Cells Induced by RANKL}

We used recombinant mouse RANKL to induce osteoclast differentiation in RAW 264.7 cells. As shown in Figure 10A, multinucleated TRAP-positive cells were observed among RAW 264.7 cells with an increase in the bone-absorptive capacity measured by the Osteo Plate Assay. We also found that treatment with AcSDKP prevented osteoclast differentiation and inhibited the bone-absorptive capacity of RANKL-treated RAW 264.7 cells.

Next, we examined the effect of Ac-SDKP on osteoclast-related signaling pathways. As shown in Figure 10B, Ac-SDKP inhibited the major regulators of RANKL signaling (including OC-STAMP, AP-1, NFATC1, and NF$\kappa \mathrm{B})$ in a dose-dependent manner. Ac-SDKP treatment also attenuated the increased levels of OC-STAMP, AP-1, NFATC1, and NF- $\mathrm{KB}$ induced by RANKL (Figure 10C). 
A

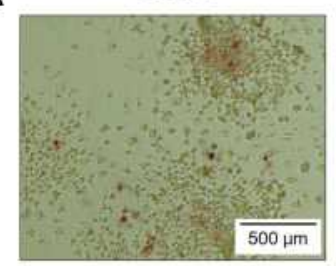

$\mathrm{SiO}_{2}$

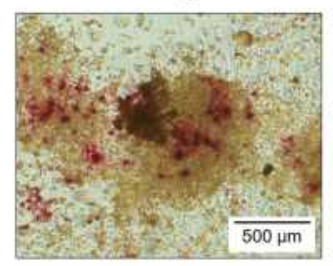

OC-STAMP

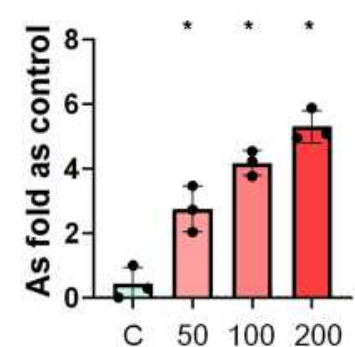

(One way ANOVA,

$F=16.083$ )

C

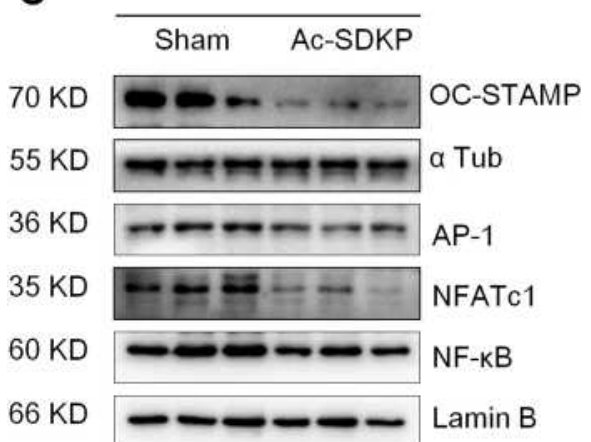

Ac-SDKP

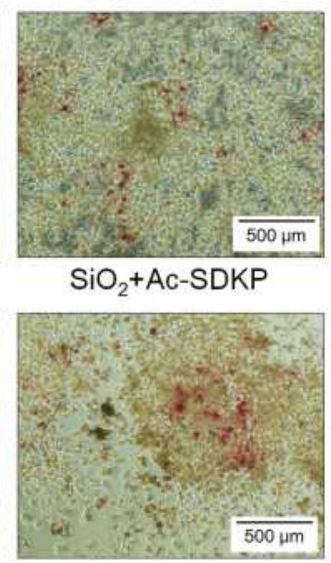

B

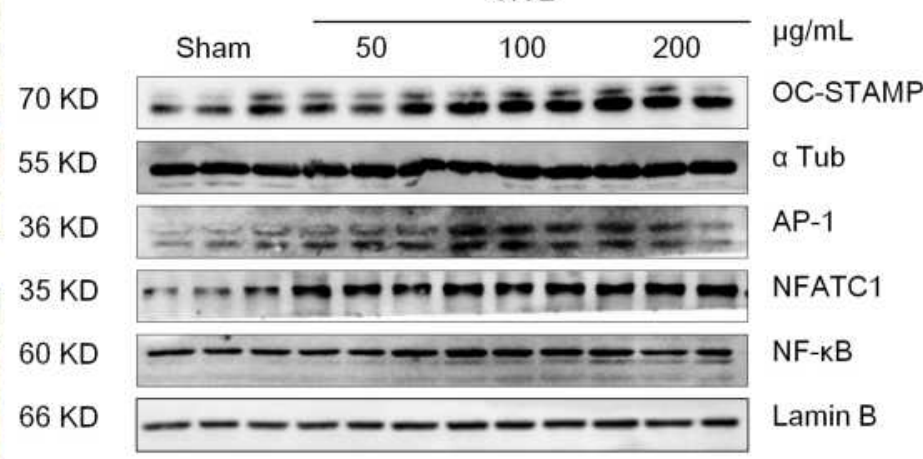

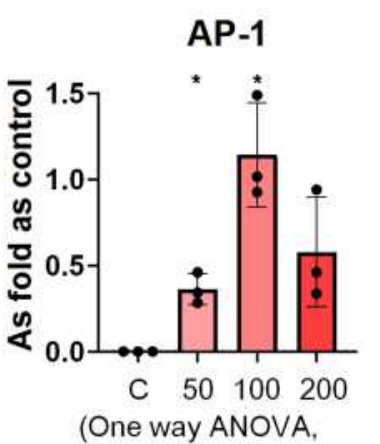

$F=22.351$ )

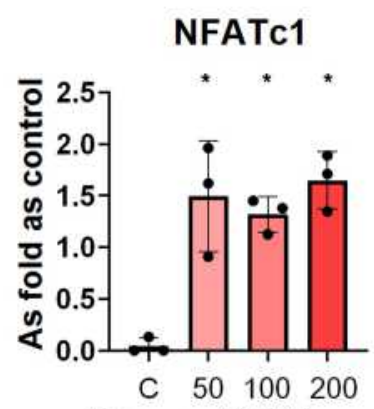

(One way ANOVA, $F=18.159$ )

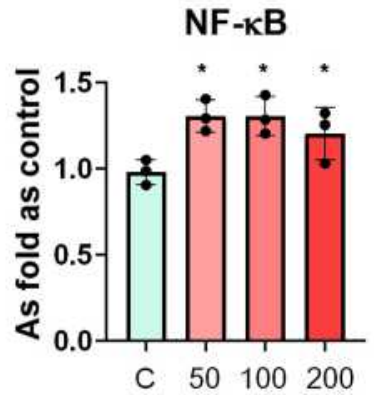

(One way ANOVA, $F=9.938$ )

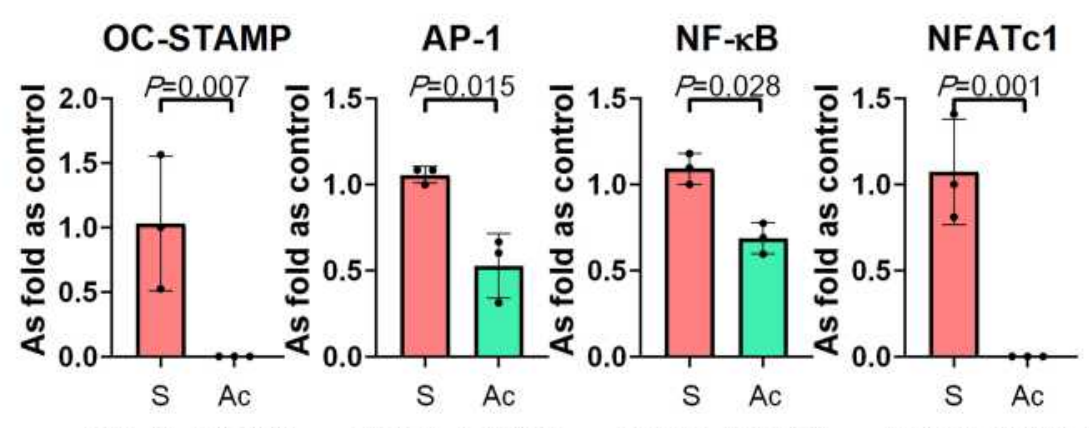

(T test, $t=5.129$ )

( $\mathrm{T}$ test, $t=4.083$ )

( $\mathrm{T}$ test, $t=3.379$ )

( $\mathrm{T}$ test, $t=8.861$ )

Figure 6 Ac-SDKP reduces TRAP-positive cells and RANKL signaling in RAW 264.7 cells treated with $\mathrm{SiO}_{2}$. (A) TRAP staining, Bar= $500 \mu \mathrm{m}$; (B) Levels of OC-STAMP, API, NFATcl, and NF-KB in RAW 264.7 cells treated with 50, 100, and $200 \mu \mathrm{g} / \mathrm{mL}$ silica measured by Western blotting. *Compared with control group, $P<0.05$. Data are presented as the mean \pm SD. $n=3$ per group. (C) Protein expression of OC-STAMP, AP-I, NFATcl, and NF-kB in RAW 264.7 cells treated with $\mathrm{SiO}_{2}$ and Ac-SDKP or not. Data are presented as the mean \pm SD. $n=3$ per group.

\section{Discussion}

The main finding in this study is that chronic inhalation of silica into the lungs of rats leads to activation of lung macrophages and bone osteoclasts via RANKL and TLR4 signaling. Most importantly, we found that Ac-SDKP treatment attenuates silica-induced lung inflammation to maintain the elastin level in lungs and to inhibit osteoclast differentiation in bone (Figure 11).
We assumed that TLR4 may promote lung inflammation in silica-treated rats. Activation of TLR4 uses MyD88 to phosphorylate $\mathrm{I} \kappa \mathrm{B}$ and initiate the NF- $\mathrm{KB}$ signaling pathway, which results in production of proinflammatory cytokines. ${ }^{32}$ TLR4-dependent fibroblast activation is reportedly a major driver of persistent organ fibrosis in systemic sclerosis. ${ }^{33}$ However, some studies have shown that expression of TLR4 on type 2 alveolar epithelial cells is important for the renewal of these cells and repair of 


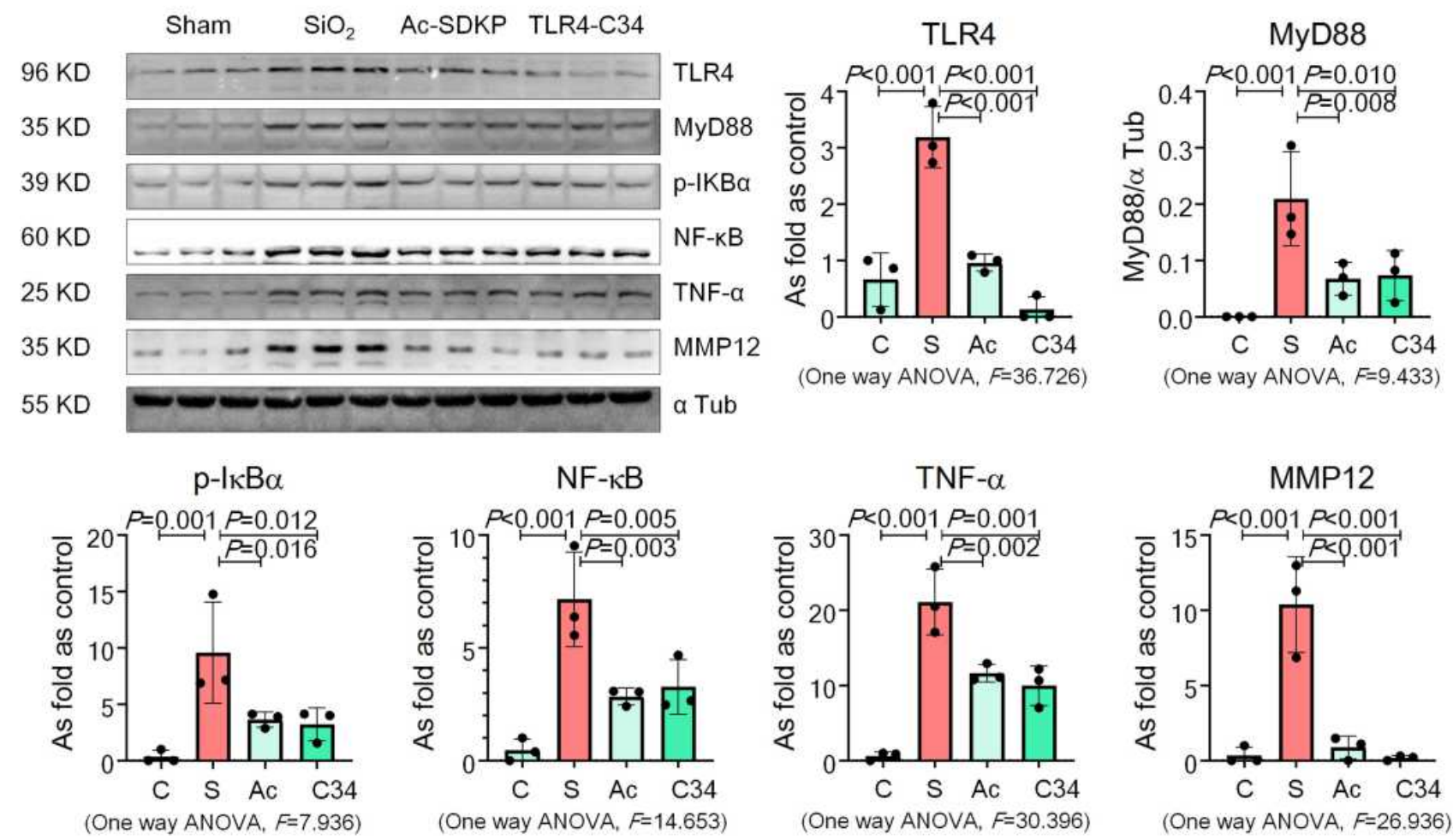

Figure 7 Ac-SDKP reduces TLR4 signaling and MMP- 12 in silica-treated macrophages. Levels of TLR4, MyD88, p-IKB $\alpha$, TNF- $\alpha$, and MMP-12 in RAW 264.7 cells measured by Western blotting. Data are presented as the mean \pm SD. $n=3$ per group.

lung injury, limiting the extent of fibrosis. ${ }^{34,35}$ In silicatreated U937-differentiated macrophages, TLR4 and the related MyD88 pathway are activated and release IL-1 $\beta$, IL-6, and TNF- $\alpha$, which suggests a pro-inflammatory effect of TLR4 on silicosis. ${ }^{9}$ These findings suggest that the different roles of TLR4 are related to different cell types, disease types, and stages of lung fibrosis. In the present study, we found that TLR4-related pathways were increased in silicotic rats and silica-treated macrophages. Therefore, we speculate that silica-induced lung inflammation is the major cause of systemic inflammation that may disturb extrapulmonary organs in rats during long-term silica inhalation. These results partially and presumably explain why air particulate matter is associated with bone
A

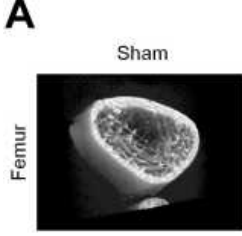

B

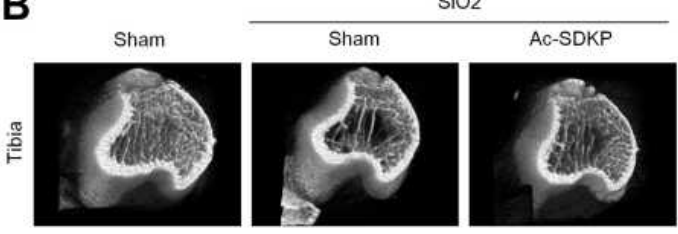

$\mathrm{SiO} 2$
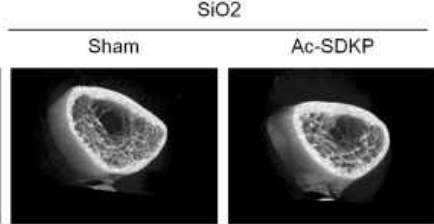

02

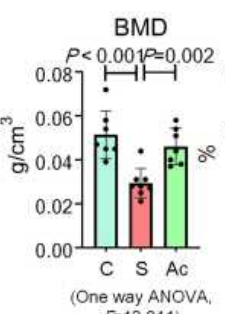

(One way ANOV
$F=13.011$ )
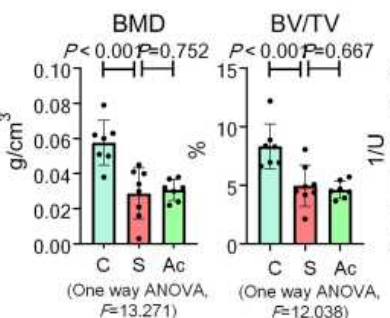

BVITV
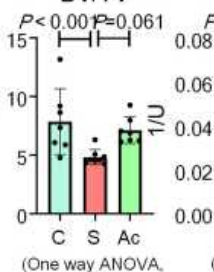

$\underset{F=6.192)}{ }$

(One way ANOVA

$F=12.268$ )
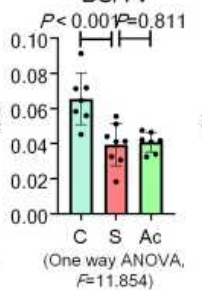
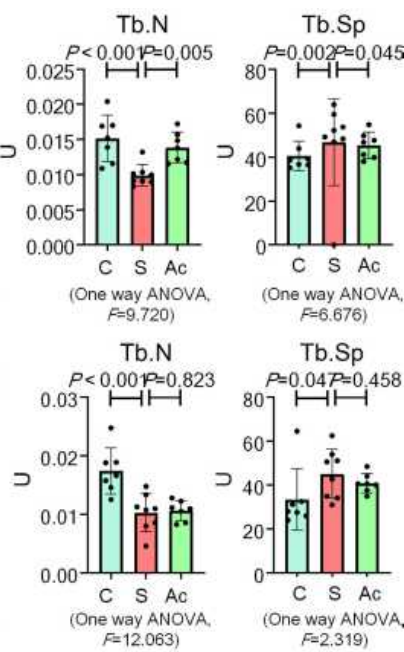

Figure 8 Ac-SDKP inhibits the decrease in BMD in the femur. (A) Bone microstructure in the femur; (B) Bone microstructure in the tibia. 
A
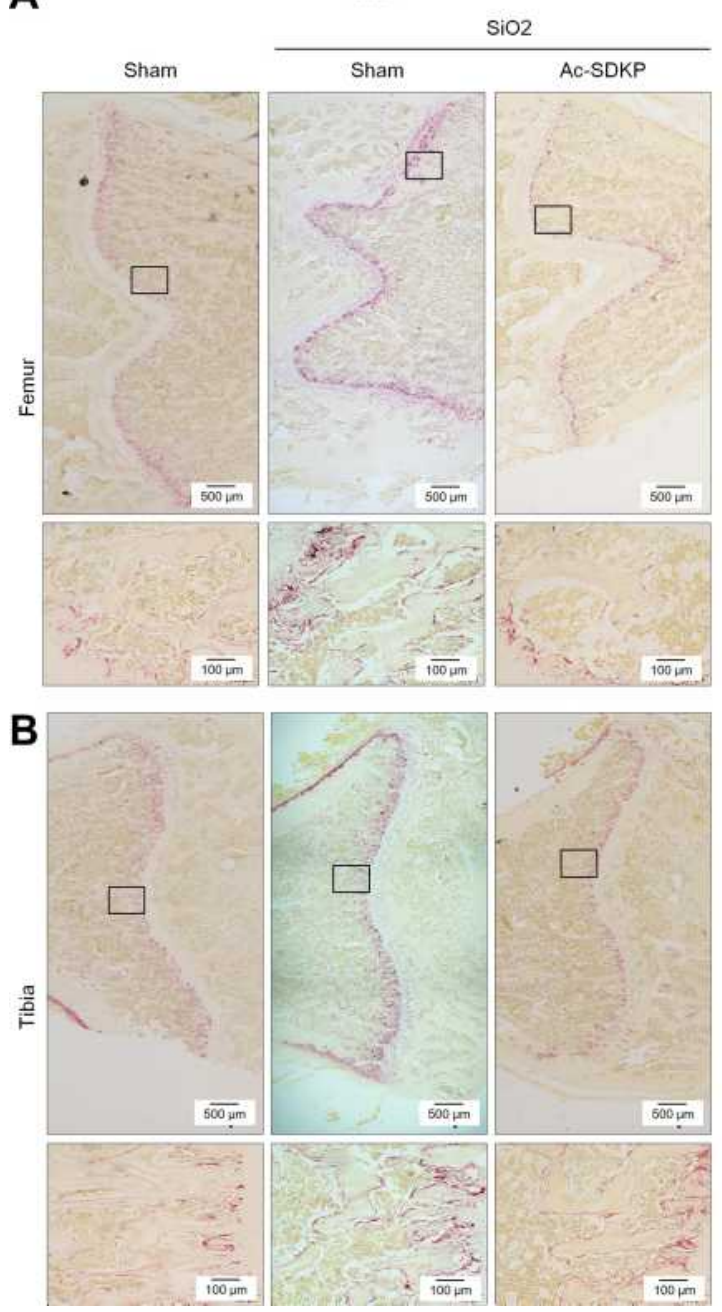
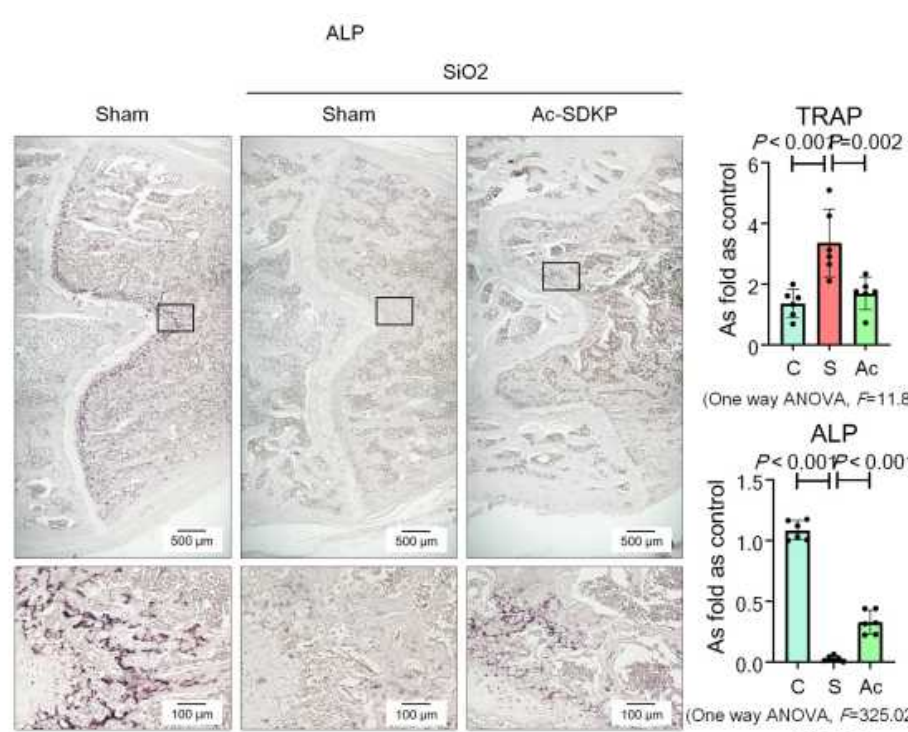

(One way ANOVA, $F=11.839$ ) ALP
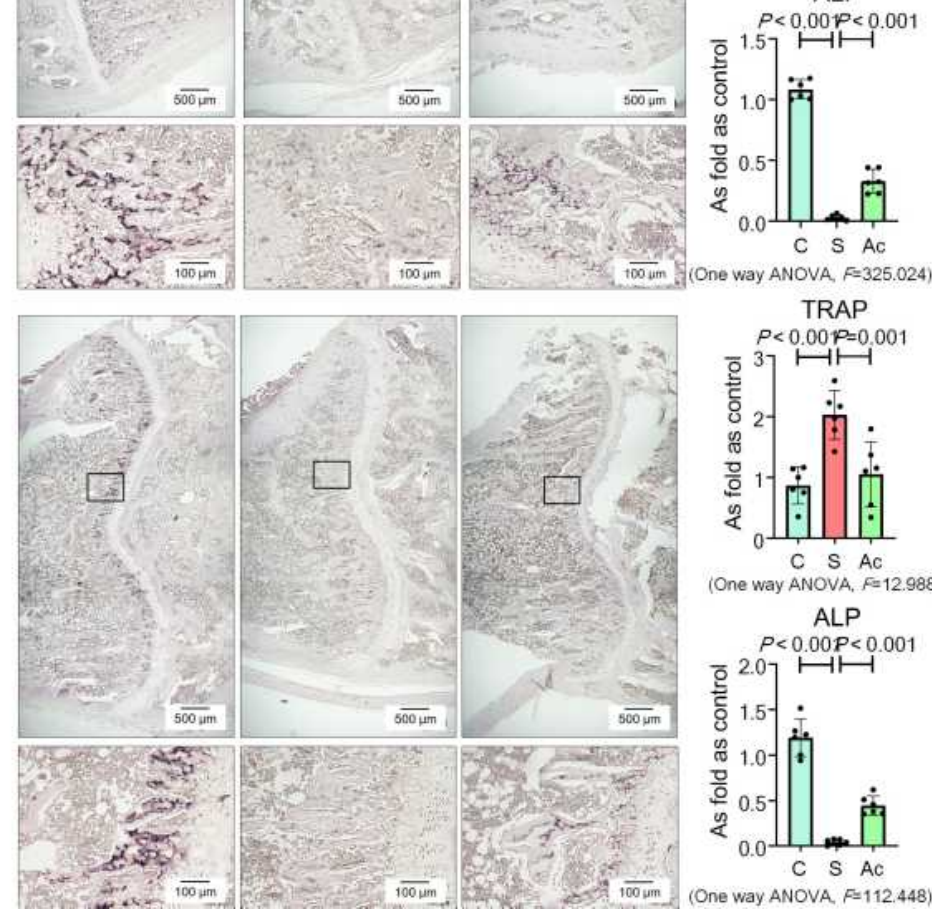

TRAP

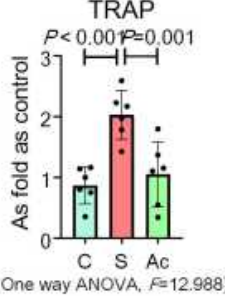

ALP

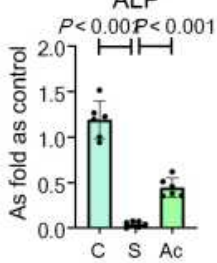

Figure 9 Ac-SDKP inhibits osteoclast differentiation in silicotic rats. (A) TRAP staining and ALP staining in the femur, Bar= $500 \mu \mathrm{m}$ and $100 \mu \mathrm{m} ;$ (B) TRAP staining and ALP staining in the tibia, Bar $=500 \mu \mathrm{m}$ and $100 \mu \mathrm{m}$.

loss and fracture risk and aggravates the severity of osteoarthritis. $^{36,37}$

Increased levels of RANKL have been documented in cystic fibrosis-related bone disease, ${ }^{38}$ adjuvant arthritisinterstitial lung disease, ${ }^{39}$ and metastasis of lung cancer to bone. ${ }^{40}$ The increased levels of RANKL in these studies suggest osteoclast activation with lung disease. In the present study, TRAP-positive macrophages and the RANKL signaling pathway, including RANKL, OCSTAMP, AP-1, NFATc1, and NF- $\mathrm{KB}$, were increased in the lungs of rats exposed to silica. These results indicate that inhalation of silica causes pulmonary macrophages to exhibit some features of osteoclasts. Although silica did not induce the transformation of NR8383 or RAW 264.7 cells into multinucleated giant cells, silica increased the proteolytic activity of macrophages with a TRAP-positive and high MMP-12 expression phenotype that potentially degrades elastin and promotes lung fibrosis formation.

Ac-SDKP is a naturally occurring immunomodulatory and pro-angiogenic peptide mainly released from its precursor thymosin $\beta 4$, whereas Ac-SDKP degradation is primarily carried out by angiotensin converting enzyme. Basal endogenous Ac-SDKP is essential for inhibiting excess collagen synthesis in normal physiological conditions. ${ }^{16}$ The anti-inflammatory activity of Ac-SDKP is partially attributed to inhibition of the differentiation of bone marrow stem cells into macrophages, macrophage activation and migration, and the release of TNF- $\alpha$ by activated macrophages. ${ }^{41}$ Ac-SDKP prevents macrophage infiltration in rodent models of angiotensin II-induced hypertension, ${ }^{42}$ traumatic brain injury, ${ }^{43}$ radiationinduced cardiotoxicity, ${ }^{44}$ myocardial infarction, ${ }^{19}$ and 


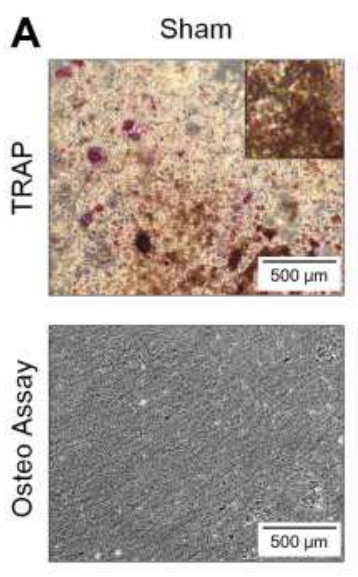

B
RANKL
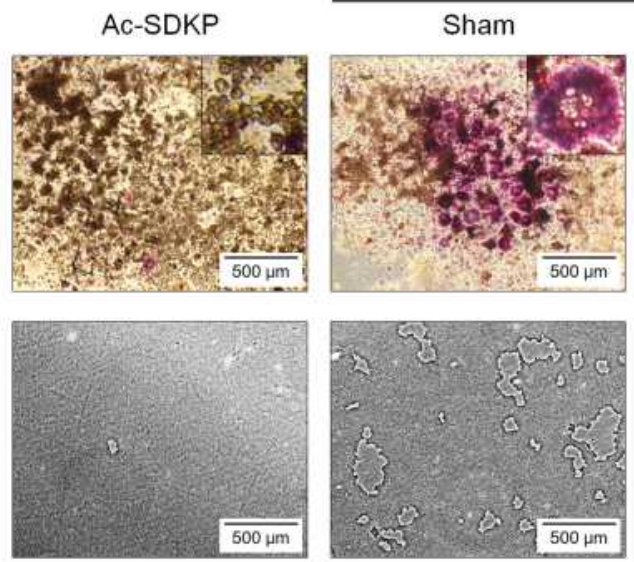
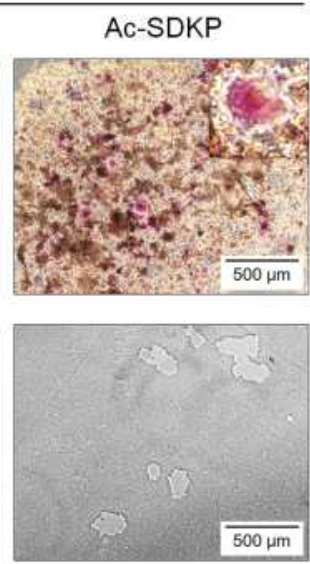

OC-STAMP

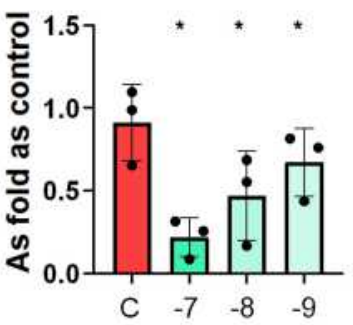

(One way ANOVA, $F=5.739$ )

NFATC1
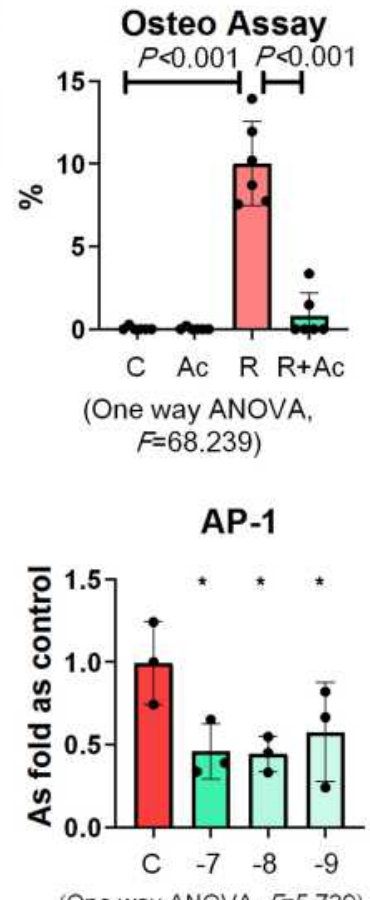

(One way ANOVA, $F=5.739$ )

NF-kB

$60 \mathrm{KD}$

$66 \mathrm{KD}$

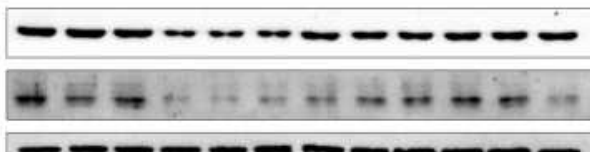

NFATC1

$N F-\kappa B$

Lamin B

C

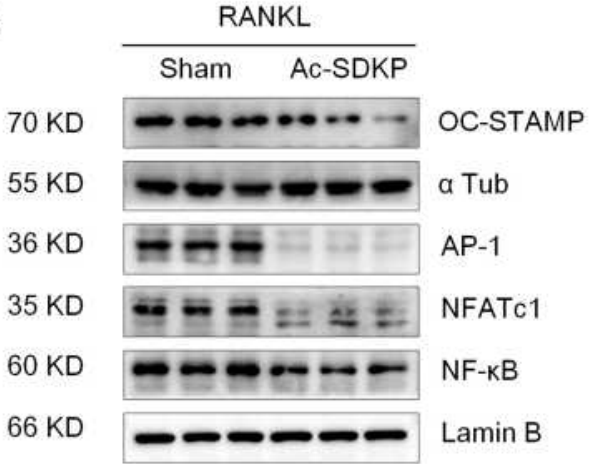

OC-STAMP

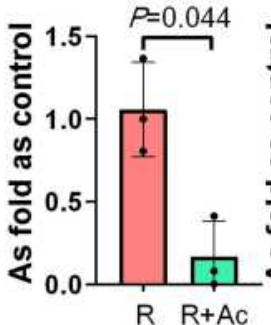

( $T$ test, $=2.905$ )

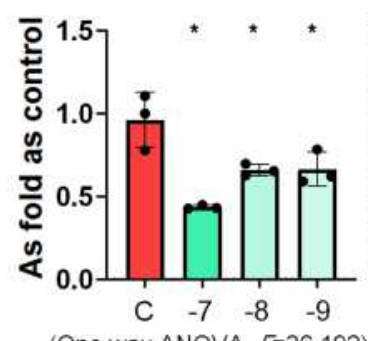

(One way ANOVA, $F=36.192$ )

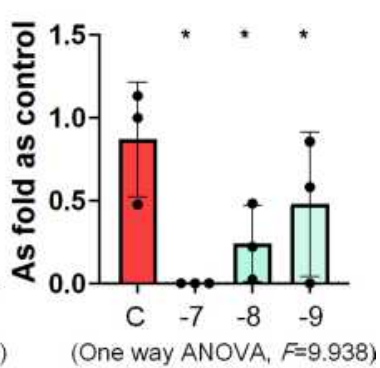

Figure 10 Ac-SDKP inhibits osteoclast differentiation in RAW 264.7 cells treated with RANKL. (A) TRAP staining and Osteo assay, Bar=500 $\mu$ m. (B) Protein expression of OC-STAMP, AP-I, NFATcI, and NF-אB in RAW 264.7 cells treated with Ac-SDKP in different dose measured by Western blotting. *Compared with control group, P<0.05. Data are presented as the mean \pm SD. $n=3$ per group. (C) Protein expression of OC-STAMP, AP-I, NFATcI, and NF- $k B$ in RAW 264.7 cells treated with RANKL and AcSDKP or not. Data are presented as the mean \pm SD. $n=3$ per group.

renal fibrosis. ${ }^{20}$ In the present study, treatment with AcSDKP inhibited lung inflammation via the RANKL and TLR4 signaling pathways in silicotic rats and reduced lung macrophages that expressed TRAP and MMP-12 to maintain the elastin level. Furthermore, Ac-SDKP inhibited osteoclastogenesis in RAW 264.7 cells induced by 


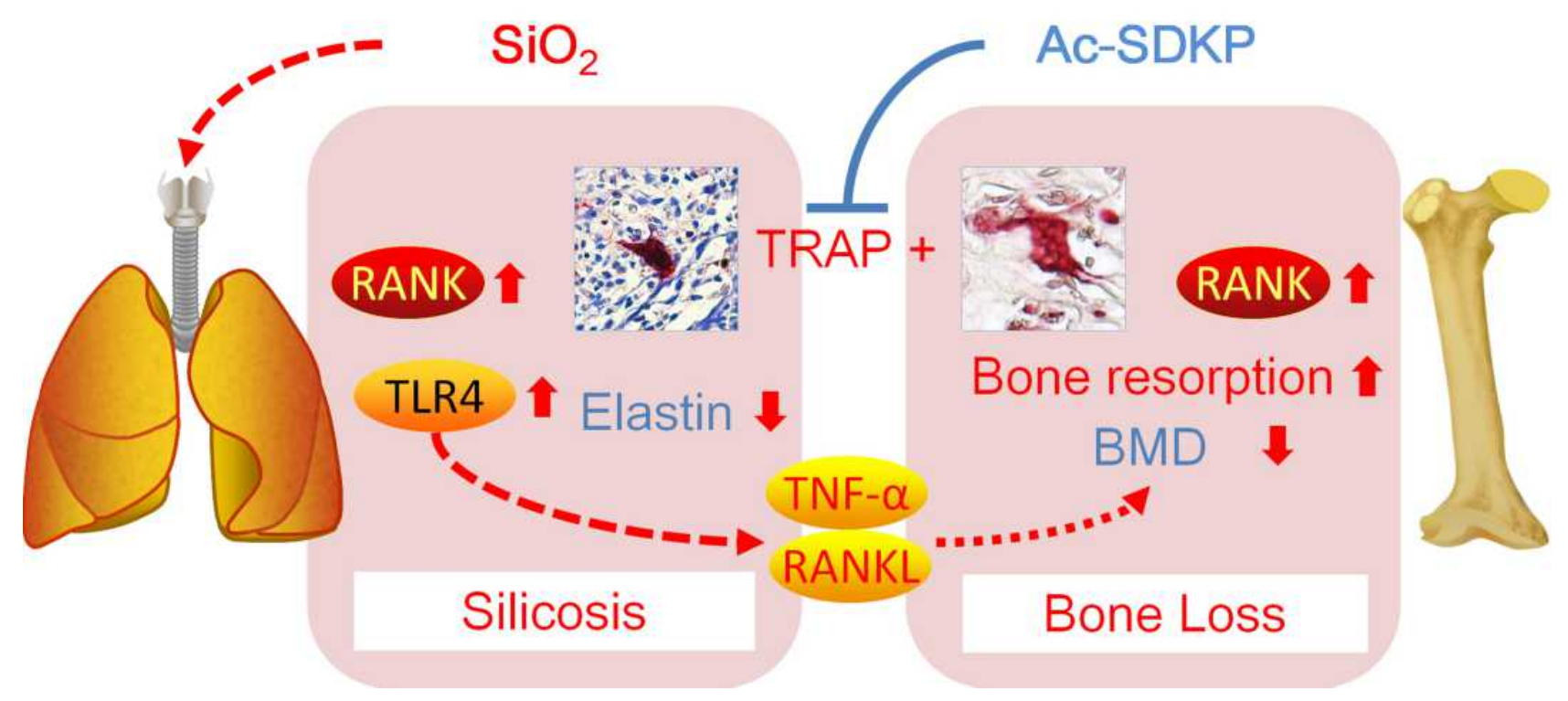

Figure II Ac-SDKP attenuates activation of lung macrophages and bone osteoclasts in rats exposed to silica.

RANKL. In vivo experiments showed that Ac-SDKP decreased the high level of osteoclast activation and sustained the osteoblast level, although the reduction in tibial BMD was not reversed by Ac-SDKP treatment.

Our study has some limitations. First, although most patients with silicosis are male, it is important to note that age and sex are also major risk factors for bone loss and osteoporosis. Second, the effects of Ac-SDKP on osteoclast differentiation should be verified in primary cells derived from bone marrow or circulating monocytes. With respect to the different effects of Ac-SDKP on the femur and tibia, we speculated that bone loss in the proximal tibia occurs earlier and is more severe than that in the femur, which has been documented in ovariectomized rats. ${ }^{45}$ Although the proximal tibia and the femur are the main fracture sites in humans and are clinically relevant, ${ }^{45}$ other bone sites including the proximal femur and lumbar vertebrae should be analyzed to more thoroughly explore the effects of silica on bone loss. Further investigations are warranted to consider and amend these limitations.

\section{Conclusions}

In summary, we demonstrated that silica inhalation caused lung macrophage and osteoclast activation via a common signaling pathway. Furthermore, Ac-SDKP stabilized lung and bone homeostasis by inhibiting activation of macrophages and osteoblasts induced by inhaled silica.

\section{Acknowledgment}

We thank Mitchell Arico and Angela Morben, DVM, ELS, from Liwen Bianji, Edanz Editing China, for editing the English text of a draft of this manuscript.

\section{Author Contributions}

All authors contributed to the data analysis and the drafting or revising of the article. All authors have also agreed on the journal to which the article will be submitted, gave final approval of the version to be published, and agree to be accountable for all aspects of the work.

\section{Funding}

This work was funded by the National Natural Science Foundation of China (No. 81972988), the National Natural Science Foundation of Hebei Province (H2020209052), the Science and Technology Research Project of Hebei Education Department (No. ZD2019077), the Science and Technology Plan Project of Tangshan City (20130206b), and the Open Fund of Key Laboratory of Functional and Clinical Translational Medicine of Xiamen Medical College (XMMC-FCTM201902). 


\section{Disclosure}

The authors declare that they have no competing interests.

\section{References}

1. Lee S, Hayashi H, Mastuzaki H, Kumagai-Takei N, Otsuki T. Silicosis and autoimmunity. Curr Opin Allergy Clin Immunol. 2017;17(2):78-84. doi:10.1097/ACI.0000000000000350

2. Rees D, Murray J. Silica, silicosis and tuberculosis. Int J Tuberc Lung Dis. 2007;11(5):474-484.

3. Pollard KM. Silica, silicosis, and autoimmunity. Front Immunol. 2016;7:97. doi:10.3389/fimmu.2016.00097

4. Sato T, Shimosato T, Klinman DM. Silicosis and lung cancer: current perspectives. Lung Cancer (Auckl). 2018;9:91-101. doi:10.2147/ LCTT.S156376

5. Millerick-May ML, Schrauben S, Reilly MJ, Rosenman KD. Silicosis and chronic renal disease. Am J Ind Med. 2015;58(7):730-736. doi:10.1002/ajim.22465

6. Yildizgoren MT, Ozis TN, Baki AE, et al. Evaluation of bone mineral density and 25-hydroxyvitamin D levels in subjects with silica exposure. Environ Health Prev Med. 2016;21(3):149-153. doi:10.1007/s12199-016-0509-3

7. Arens AM, Barr B, Puchalski SM, et al. Osteoporosis associated with pulmonary silicosis in an equine bone fragility syndrome. Vet Pathol. 2011;48(3):593-615. doi:10.1177/0300985810385151

8. Hui Z, Dingjie X, Yuan Y, et al. Silicosis decreases bone mineral density in rats. Toxicol Appl Pharmacol. 2018;348:117-122. doi:10.1016/j.taap.2018.04.023

9. Chan JYW, Tsui JCC, Law PTW, et al. Regulation of TLR4 in silica-induced inflammation: an underlying mechanism of silicosis. Int J Med Sci. 2018;15(10):986-991. doi:10.7150/ijms.24715

10. Pereira M, Petretto E, Gordon S, et al. Common signalling pathways in macrophage and osteoclast multinucleation. J Cell Sci. 2018;131 (11):jcs216267. doi:10.1242/jcs.216267

11. Walsh MC, Choi Y. Biology of the RANKL-RANK-OPG system in immunity, bone, and beyond. Front Immunol. 2014;5:511. doi:10.3389/fimmu.2014.00511

12. Park JH, Lee NK, Lee SY. Current understanding of RANK signaling in osteoclast differentiation and maturation. Mol Cells. 2017;40 (10):706-713. doi:10.14348/molcells.2017.0225

13. Boorsma CE, van der Veen TA, Putri KSS, et al. A potent tartrate resistant acid phosphatase inhibitor to study the function of TRAP in alveolar macrophages. Sci Rep. 2017;7(1):12570. doi:10.1038/ s41598-017-12623-w

14. Igari K, Kelly MJ, Yamanouchi D. Cigarette smoke extract activates tartrate-resistant acid phosphatase-positive macrophage. J Vasc Res. 2019;56(3):139-151. doi:10.1159/000498893

15. Tsuboi H, Matsui Y, Hayashida K, et al. Tartrate resistant acid phosphatase (TRAP) positive cells in rheumatoid synovium may induce the destruction of articular cartilage. Ann Rheum Dis. 2003;62(3):196-203. doi:10.1136/ard.62.3.196

16. Kumar N, Yin C. The anti-inflammatory peptide Ac-SDKP: synthesis, role in ACE inhibition, and its therapeutic potential in hypertension and cardiovascular diseases. Pharmacol Res. 2018;134:268-279. doi:10.1016/j.phrs.2018.07.006

17. Zhang L, Xu D, Li Q, et al. N-acetyl-seryl-aspartyl-lysyl-proline (Ac-SDKP) attenuates silicotic fibrosis by suppressing apoptosis of alveolar type II epithelial cells via mediation of endoplasmic reticulum stress. Toxicol Appl Pharmacol. 2018;350:1-10. doi:10.1016/j. taap.2018.04.025

18. Shifeng L, Hong X, Xue Y, et al. Ac-SDKP increases alpha-TAT 1 and promotes the apoptosis in lung fibroblasts and epithelial cells double-stimulated with TGF-beta1 and silica. Toxicol Appl Pharmacol. 2019;369:17-29. doi:10.1016/j.taap.2019.02.015
19. Ma X, Yuan Y, Zhang Z, Zhang Y, Li M. An analog of Ac-SDKP improves heart functions after myocardial infarction by suppressing alternative activation (M2) of macrophages. Int J Cardiol. 2014;175 (2):376-378. doi:10.1016/j.ijcard.2014.05.016

20. Chan GC, Yiu WH, Wu HJ, et al. N-acetyl-seryl-aspartyl-lysylproline alleviates renal fibrosis induced by unilateral ureteric obstruction in BALB/C mice. Mediators Inflamm. 2015;2015:283123. doi: $10.1155 / 2015 / 283123$

21. Worlein JM, Baker K, Bloomsmith M, Coleman K, Koban TL. The eighth edition of the guide for the care and use of laboratory animals (2011); implications for behavioral management. Am J Primatol. 2011;73:98.

22. Chen Y, Xu D, Yao J, et al. Inhibition of miR-155-5p exerts anti-fibrotic effects in silicotic mice by regulating meprin alpha. Mol Ther Nucleic Acids. 2020;19:350-360. doi:10.1016/j. omtn.2019.11.018

23. Song C, Yang X, Lei Y, et al. Evaluation of efficacy on RANKL induced osteoclast from RAW264.7 cells. J Cell Physiol. 2019;234 (7):11969-11975. doi:10.1002/jcp.27852

24. Deng H, Xu H, Zhang X, et al. Protective effect of Ac-SDKP on alveolar epithelial cells through inhibition of EMT via TGF-beta1/ ROCK1 pathway in silicosis in rat. Toxicol Appl Pharmacol. 2016;294:1-10. doi:10.1016/j.taap.2016.01.010

25. Neal MD, Jia H, Eyer B, et al. Discovery and validation of a new class of small molecule toll-like receptor 4 (TLR4) inhibitors. PLoS One. 2013;8(6):e65779. doi:10.1371/journal.pone.0065779

26. Zhang B, Xu H, Zhang Y, et al. Targeting the RAS axis alleviates silicotic fibrosis and Ang II-induced myofibroblast differentiation via inhibition of the hedgehog signaling pathway. Toxicol Lett. 2019;313:30-41. doi:10.1016/j.toxlet.2019.05.023

27. Zhou L, Le Y, Tian J, et al. Cigarette smoke-induced RANKL expression enhances MMP-9 production by alveolar macrophages. Int J Chron Obstruct Pulmon Dis. 2019;14:81-91. doi:10.2147/ COPD.S190023

28. Shichino S, Abe J, Ueha S, et al. Reduced supply of monocyte-derived macrophages leads to a transition from nodular to diffuse lesions and tissue cell activation in silica-induced pulmonary fibrosis in mice. Am J Pathol. 2015;185(11):2923-2938. doi:10.1016/j.ajpath.2015.07.013

29. Zhou Z, Jiang R, Yang X, et al. circRNA mediates silica-induced macrophage activation via HECTD1/ZC3H12A-dependent ubiquitination. Theranostics. 2018;8(2):575-592. doi:10.7150/ thno. 21648

30. Carneiro PJ, Clevelario AL, Padilha GA, et al. Bosutinib therapy ameliorates lung inflammation and fibrosis in experimental silicosis. Front Physiol. 2017;8:159. doi:10.3389/fphys.2017.00159

31. Li X, Hu Y, Jin Z, Jiang H, Wen J. Silica-induced TNF- $\alpha$ and TGF- $\beta$ 1 expression in RAW264.7 cells are dependent on Src-ERK/AP-1 pathways. Toxicol Mech Methods. 2009;19(1):51-58. doi:10.1080/ 15376510802354201

32. Kuzmich NN, Sivak KV, Chubarev VN, et al. TLR4 signaling pathway modulators as potential therapeutics in inflammation and sepsis. Vaccines (Basel). 2017;5(4). doi:10.3390/vaccines5040034

33. Bhattacharyya S, Wang W, Qin W, et al. TLR4-dependent fibroblast activation drives persistent organ fibrosis in skin and lung. JCI Insight. 2018;3(13). doi:10.1172/jci.insight.98850

34. Liang J, Zhang Y, Xie T, et al. Hyaluronan and TLR4 promote surfactant-protein-C-positive alveolar progenitor cell renewal and prevent severe pulmonary fibrosis in mice. Nat Med. 2016;22 (11): 1285-1293. doi:10.1038/nm.4192

35. Yang HZ, Wang JP, Mi S, et al. TLR4 activity is required in the resolution of pulmonary inflammation and fibrosis after acute and chronic lung injury. Am J Pathol. 2012;180(1):275-292. doi:10.1016/ j.ajpath.2011.09.019 
36. Prada D, Zhong J, Colicino E, et al. Association of air particulate pollution with bone loss over time and bone fracture risk: analysis of data from two independent studies. Lancet Planet Health. 2017;1(8): e337-e347. doi:10.1016/S2542-5196(17)30136-5

37. Peng KT, Liu JF, Chiang YC, et al. Particulate matter exposure aggravates osteoarthritis severity. Clin Sci (Lond). 2019;133 (21):2171-2187. doi:10.1042/CS20190458

38. Gensburger D, Boutroy S, Chapurlat R, et al. Reduced bone volumetric density and weak correlation between infection and bone markers in cystic fibrosis adult patients. Osteoporos Int. 2016;27 (9):2803-2813. doi:10.1007/s00198-016-3612-9

39. Yang G, Lyu L, Wang X, et al. Systemic treatment with resveratrol alleviates adjuvant arthritis-interstitial lung disease in rats via modulation of JAK/STAT/RANKL signaling pathway. Pulm Pharmacol Ther. 2019;56:69-74. doi:10.1016/j.pupt.2019.03.011

40. Wang M, Chao CC, Chen PC, et al. Thrombospondin enhances RANKL-dependent osteoclastogenesis and facilitates lung cancer bone metastasis. Biochem Pharmacol. 2019;166:23-32. doi:10.10 16/j.bcp.2019.05.005
41. Dadar M, Shahali Y, Chakraborty S, et al. Antiinflammatory peptides: current knowledge and promising prospects. Inflamm Res. 2019;68 (2):125-145. doi:10.1007/s00011-018-1208-x

42. Gonzalez GE, Rhaleb NE, Nakagawa P, et al. N-acetyl-seryl-aspartyllysyl-proline reduces cardiac collagen cross-linking and inflammation in angiotensin II-induced hypertensive rats. Clin Sci (Lond). 2014;126(1):85-94. doi:10.1042/CS20120619

43. Zhang Y, Zhang ZG, Chopp M, et al. Treatment of traumatic brain injury in rats with $\mathrm{N}$-acetyl-seryl-aspartyl-lysyl-proline. J Neurosurg. 2017;126(3):782-795. doi:10.3171/2016.3.JNS152699

44. Sharma UC, Sonkawade SD, Spernyak JA, et al. A small peptide Ac-SDKP inhibits radiation-induced cardiomyopathy. Circ Heart Fail. 2018;11(8):e004867. doi:10.1161/CIRCHEARTFAILURE. 117.004867

45. Yousefzadeh N, Kashfi K, Jeddi S, Ghasemi A. Ovariectomized rat model of osteoporosis: a practical guide. EXCLI J. 2020;19:89-107. doi:10.17179/excli2019-1990
Journal of Inflammation Research

\section{Publish your work in this journal}

The Journal of Inflammation Research is an international, peerreviewed open-access journal that welcomes laboratory and clinical findings on the molecular basis, cell biology and pharmacology of inflammation including original research, reviews, symposium reports, hypothesis formation and commentaries on: acute/chronic inflammation; mediators of inflammation; cellular processes; molecular
Dovepress

mechanisms; pharmacology and novel anti-inflammatory drugs; clinical conditions involving inflammation. The manuscript management system is completely online and includes a very quick and fair peerreview system. Visit http://www.dovepress.com/testimonials.php to read real quotes from published authors. 